\title{
Relation between the metastability of $E L 2$ and the photosensitivity of local vibrational modes in semi-insulating GaAs
}

\author{
C. Y. Song \\ Institute of Semiconductors, Chinese Academy of Sciences, P.O. Box 912, 100083, Beijing, China \\ B. Pajot \\ Groupe de Physique des Solides, Tour 23, Université Denis Diderot, 2 place Jussieu, 75251 Paris Cedex 05, France \\ W. K. Ge \\ Department of Physics, The Hong Kong University of Science \& Technology, Clear Water Bay, Kowloon, Hong Kong \\ D. S. Jiang \\ Institute of Semiconductors, Chinese Academy of Sciences, P.O. Box 912, 100083, Beijing, China
}

(Received 15 March 1995)

\begin{abstract}
Recent infrared spectroscpic observations of local vibrational mode absorptions have revealed a number of photosensitive centers in semi-insulating GaAs. They include $\left(\mathrm{O} V_{\mathrm{As}}\right)$ center which has three modes at $730 \mathrm{~cm}^{-1}(A), 715 \mathrm{~cm}^{-1}(B)$, and $714 \mathrm{~cm}^{-1}(C)$, respectively, a suggested $\mathrm{NH}$ center related to a line at $983 \mathrm{~cm}^{-1}\left(X_{1}\right)$, and centers related to hydrogen, such as (H-O) or (H-N) bonds, corresponding to a group of peaks in the region of $2900-3500 \mathrm{~cm}^{-1}$. The photosensitivity of various local vibration centers was observed to have similar time dependence under near-infrared illumination and was suggested to be due to their charge-state interconversion. Mainly described in this work is the effect of the $1.25-\mathrm{eV}$ illumination. It is confirmed that this photoinduced kinetic process results from both electron capture and hole capture, which are closely related to the photoionization behavior and metastability of the $E L 2$ center.
\end{abstract}

\section{INTRODUCTION}

In connection with the metastability of the deep defect $E L 2$, the photosensitivity of local vibrational modes (LVM's) related to metastable centers or to centers with more than one electronic charge state has been reported in recent years in semi-insulating (SI) GaAs. ${ }^{1-5}$ This shows that LVM absorption can also bring information on the electronic charge state of deep defects and on the location of their levels in the band gap. The first photosensitive defect whose LVM's have been studied is the oxygen-vacancy center. From isotope effects, absorption under uniaxial stress, and photoinduced absorption, ${ }^{2,4-6}$ this center has been established to be an off-center $O$ atom bonded to two $\mathrm{Ga}$ atoms. Alternatively, it can also be considered as an $\mathrm{O}$ atom trapped by an As vacancy $\left(\mathrm{O} V_{\mathrm{As}}\right)$, analogous to the well-known $A$ center in silicon. It gives rise to three vibrational features at $730 \mathrm{~cm}^{-1}$ $(A), 715 \mathrm{~cm}^{-1}(B)$, and $714 \mathrm{~cm}^{-1}\left(C\right.$, or $B^{\prime}$ in Ref. 4) due to the antisymmetric stretching motion of the $O$ atom bonded to the $\mathrm{Ga}$ atoms. The illumination- and Fermilevel-dependent conditions of observations of features $A$, $B$, and $C$ have been used to prove that $\left(O V_{A s}\right)$ is a negative- $U$ system by thermally activated decay of the LVM intensity. ${ }^{8,9}$ Other photosensitive LVM's are also observed in SI GaAs. They include two near $980 \mathrm{~cm}^{-1}$ $\left(X_{1}\right.$ and $\left.X_{2}\right)$ (Ref. 5) and more ${ }^{7}$ between 2000 and 3500 $\mathrm{cm}^{-1}$. The characteristics of these lines are listed in Table I.

It has been proposed that the photosensitive behavior of these centers in SI GaAs was related to the photoionization of $E L 2$ and to its photoquenching into the metastable state. ${ }^{3,4,10}$ We present here a correlation between the photosensitive behavior of $\left(\mathrm{O} V_{\text {As }}\right)$ and that of $\mathrm{H}$ related complexes in SI GaAs which can be well interpreted by the unusual photosensitive behavior of $E L 2$.

\section{EXPERIMENT}

We report here results on two high-resistivity GaAs samples (1 and 6) grown by the liquid-encapsulated Czochralski method from a quartz crucible. The carbon acceptor was detected by its LVM absorption at a concentration of about $1 \times 10^{15} \mathrm{~cm}^{-3}$ in these samples. The iron concentration was estimated to be $(3-4) \times 10^{13} \mathrm{~cm}^{-3}$ from the intensity ${ }^{11}$ of the $3001-\mathrm{cm}^{-1}$ electronic line of $\mathrm{Fe}^{2+}$. As far as the Fermi level can be taken as uniform in SI GaAs samples, the neutral EL 2 concentration deduced from the near IR absorption ${ }^{12}$ was 1 and $2 \times 10^{16}$ $\mathrm{cm}^{-3}$ in samples 1 and 6 , respectively. The absorption was measured with a Bomem DA3 Fourier-transform spectrometer with the samples held in an Oxford Instruments continuous flow cryostat in which the temperature could be adjusted from about $6 \mathrm{~K}$, to room temperature. The high frequency side of the spectrum was blocked by filters with threshold at 1600 or $4400 \mathrm{~cm}^{-1}(\sim 0.2$ or 0.55 $\mathrm{eV}$, respectively) placed between the FTS and the cryostat. Quasithermal equilibrium conditions (QTEC) were 
TABLE I. Characteristics of $6 \mathrm{~K}$ of the photosensitive LVM's observed. Labels are the same as those in Refs. 5 and 7. The $\mathrm{Fe}^{2+}$ lines are located at $2987.47,3001.49$, and $3001.82 \mathrm{~cm}^{-1}$. Photosensitivity (PS) or photoinduced decrease $\left(\mathbf{P I}^{-}\right)$or increase $\left(\mathbf{P I}^{+}\right)$after 1.25-eV illumination are indicated. No assignment means either a $\mathrm{NH}$ or $\mathrm{OH}$ bond.

\begin{tabular}{|c|c|c|c|c|}
\hline Label & Position $\left(\mathrm{cm}^{-1}\right)$ & FWHP $\left(\mathrm{cm}^{-1}\right)$ & Remarks & Assignment \\
\hline \multirow[t]{3}{*}{$\mathrm{C}$} & 713.76 & 0.05 & PS & $\left(\mathrm{O} V_{\mathrm{As}}\right)^{-}$ \\
\hline & 713.24 & 0.05 & PS & $\left(\mathrm{O} V_{\mathrm{As}}\right)^{-}$ \\
\hline & 714.71 & 0.05 & PS & $\left(\mathrm{O} V_{\mathrm{As}}\right)^{-}$ \\
\hline \multirow[t]{3}{*}{$B$} & 714.42 & 0.05 & PS & $\left(\mathrm{O} V_{\mathrm{As}}\right)^{2-}$ \\
\hline & 714.90 & 0.05 & PS & $\left(\mathrm{O} V_{\mathrm{As}}\right)^{2-}$ \\
\hline & 715.38 & 0.05 & PS & $\left(\mathrm{O} V_{\mathrm{As}}\right)^{2-}$ \\
\hline \multirow[t]{3}{*}{$A$} & 730.19 & 0.05 & PS & $\left(\mathrm{O} V_{\mathrm{As}}\right)^{0}$ \\
\hline & 730.68 & 0.05 & PS & $\left(\mathrm{O} V_{\mathrm{As}}\right)^{0}$ \\
\hline & 731.17 & 0.05 & $\mathbf{P}$ & $\left(\mathrm{O} V_{\mathrm{As}}\right)^{0}$ \\
\hline$X_{2}{ }^{\mathrm{a}}$ & 972.5 & 0.2 & Light induced & NH? \\
\hline$X_{1}^{a}$ & 983.33 & 0.08 & $\mathrm{PI}^{-}$ & NH? \\
\hline $1^{\prime}$ & 2791.8 & 0.4 & Light induced & NH \\
\hline 1 & 2947.35 & 0.09 & $\mathrm{PI}^{-}$ & NH \\
\hline 2 & 3015.54 & 1.0 & $\mathrm{PI}^{-}$ & \\
\hline $2^{\prime}$ & 3028.5 & 2.7 & Light induced & \\
\hline 3 & 3045.9 & 1.5 & $\mathbf{P I}^{-}$ & \\
\hline $3^{\prime}$ & 3066.3 & 1.4 & Light induced & \\
\hline 4 & 3079.22 & 0.09 & $\mathbf{P I}^{-}$ & NH? \\
\hline 6 & 3099.08 & 0.23 & Light induced & NH? \\
\hline 7 & 3108.01 & 0.10 & $\mathrm{PI}^{-}$ & \\
\hline 13 & 3296.4 & 0.5 & Light induced & $\mathrm{OH}^{-}$ \\
\hline 14 & 3299.95 & 0.13 & $\mathrm{PI}^{-}$ & $\mathrm{OH}$ \\
\hline 16 & 3425.96 & 0.08 & $\mathrm{PI}^{-}$ & \\
\hline
\end{tabular}

${ }^{\text {a }}$ Related to lines 4 and 6.

realized by cooling the sample in the dark to $6 \mathrm{~K}$ with the source off in about $40 \mathrm{~min}$ and by performing the measurement with one of the blocking filters. Additional side illumination was realized by focusing the output of a $100-W$ tungsten filament source on the sample through an interference filter centered at $1.25 \mathrm{eV}$. The absorption measurements were taken at $6 \mathrm{~K}$ with illumination off. The illumination time given for one data point is the sum of the previous illumination times.

\section{RESULTS}

\section{A. Photoinduced interconversion between $A, B$, and $C$}

In SI GaAs sample 1 , where only feature $A$ is observed under QTEC, it is found that increasing illumination time with $1.25-\mathrm{eV}$ photons produces the decrease of $A$, the growth and then decrease of $C$ and $B$, and finally the increase of $A$ which remains the only feature observed. We call this process interconversion and it is presented below in more detail.

The intensity changes of $A, B$, and $C$ in sample 1 with the illumination time shown in Fig. 1 give an overall picture of the interconversion between the different features. The triplet structure of each feature is due to the three possible combinations of the ${ }^{69} \mathrm{Ga}$ and ${ }^{71} \mathrm{Ga}$ isotopes (relative abundances are 0.6 and 0.4 , respectively). The change of the intensity of the component due to ${ }^{69} \mathrm{Ga}-\mathrm{O}-$ ${ }^{69} \mathrm{Ga}(A 1, B 1$, and $C 1)$ is plotted as a function of the il- lumination time in Figs. 2(a) and 2(b). In order to ensure accuracy while keeping a reasonable time scale, the experiment was performed with photon fluxes of 0.4 and $2.5 \times 10^{15} \mathrm{~cm}^{-2} \mathrm{~s}^{-1}$ for parts I and II, respectively. In Fig. 2(a), the intensity of $A$ is shown to decrease quickly while $C$ appears, accompanied by a subsequent turning up of $B$. After $\sim 100 \mathrm{~s}$ illumination time, $C$ reaches a maximum which is about half the initial intensity of $A$. It takes $\sim 10^{-3} \mathrm{~s}$ for $C$ to vanish ( $A$ has vanished after $\sim 200 \mathrm{~s}$ ). The intensity of $B$ reaches a plateau after about $3000 \mathrm{~s}$ with almost the same intensity as initial $A$. This intensity transfer from $A$ to $B$ via $C$ or $(A \rightarrow C \rightarrow B)$ is relatively fast compared with the one in Fig. 2(b). This latter transfer $(B \rightarrow C \rightarrow A)$ is the inverse of the first, and, to speed up the transfer, a photon flux 5.8 times larger is used. At the end of part II, $A$ has reached its initial intensity, both $C$ and $B$ vanish again, and no subsequent intensity change is observed after illumination for up to two more hours. This is the detail of the interconversion of $A, B$, and $C$, whose origin is explained below. If the photon flux used in part II is used in part I, the $(A \rightarrow C \rightarrow B$ ) transfer is reached for an illumination time shorter by a factor $\sim 5.4$, comparable with the enhancement ratio of the photon fluxes (5.8). This factor 5.4 is used to unify parts I and II using the illumination time scale of II [Fig. 2(c)], with the implicit assumption that the transfer rate is proportional to the photon flux. The photon energy of $1.25 \mathrm{eV}$ is not critical as similar results are obtained with 10-min white-light illumination. 


\section{B. The photoinduced effects on $\mathrm{Fe}^{2+}$ and on the H-related LVM's}

As reported earlier, ${ }^{7}$ many H-related LVM's are observed between 2930 and $3500 \mathrm{~cm}^{-1}$, together with the

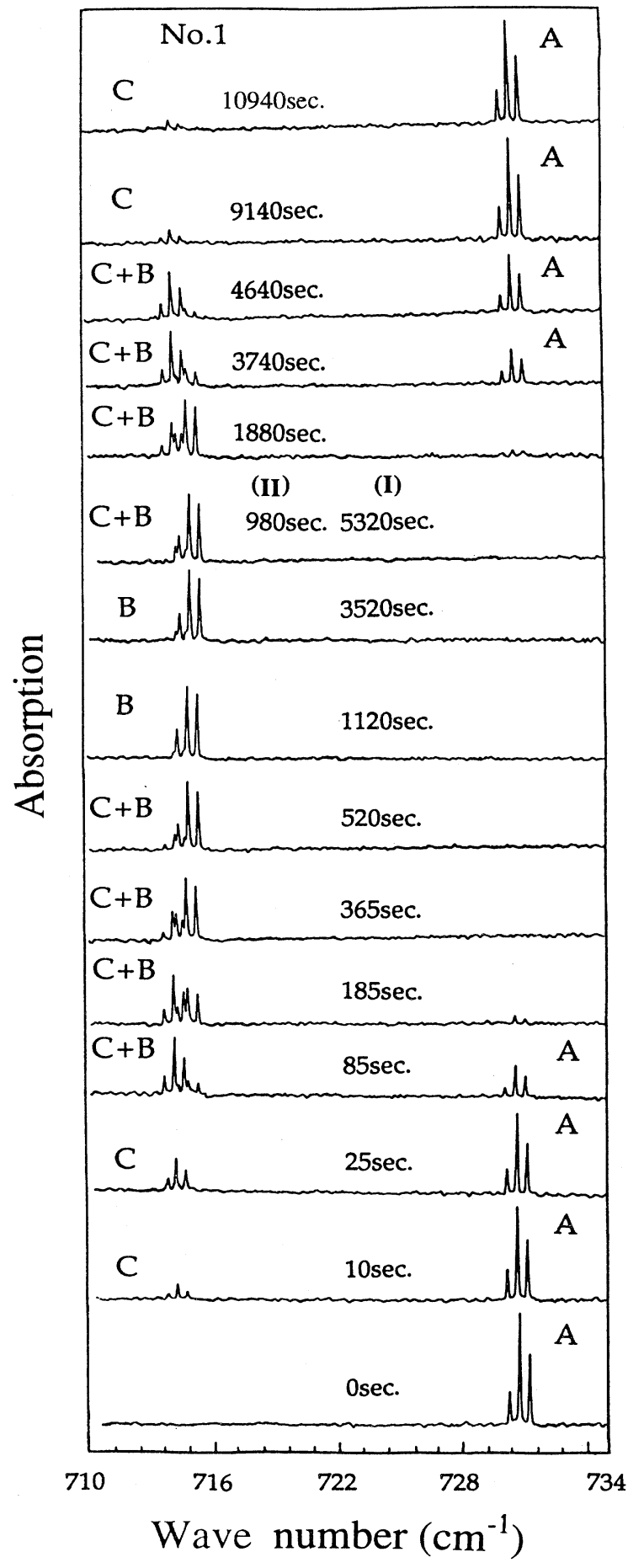

FIG. 1. Spectra showing the intensity interconversion $A \rightarrow C \rightarrow B \rightarrow A$ of local vibration modes of center $\left(\mathrm{O} V_{\mathrm{As}}\right)$ in sample 1 induced by $1.25-\mathrm{eV}$ illumination. The intensity ratio for the three modes at $5320 \mathrm{sec}$ of part I and $980 \mathrm{sec}$ of part II are basically equal. lines due to the internal electronic transitions $\left({ }^{5} E \rightarrow{ }^{5} T_{2}\right)$ of $\mathrm{Fe}^{2+}\left(2988,3001\right.$, and $\left.3002 \mathrm{~cm}^{-1}\right)$ in samples 1 and 6 . In sample 1 , many of the lines are photosensitive. Figure 3 shows difference spectra of this sample before and after illuminations for $150 \mathrm{~min}$ with $1.25 \mathrm{-eV}$ photons and for 10 min with white light. Only the photosensitive lines are revealed in these spectra. The ones rising upward are new photoinduced features (e.g., lines 6 and 13) and the ones peaking downward decrease in intensity (e.g., lines $2,4,7,14$, and 16 ) or disappear (the $\mathrm{Fe}^{2+}$ lines). In order to make a comparison with the behavior of $A, B$, and $C$, seven illumination times indicated by solid squares on the abscissa scale of Fig. 2(c) were used to study intensity changes with the illumination time. Figure 4 shows that the onset of the quenching of lines of $\mathrm{Fe}^{2+}$ and $X_{1}$ simultaneously occurs with that of the intensity decrease of lines 4 and 14, and of the appearance of lines 6 and 13. This onset occurs after the same illumination time which
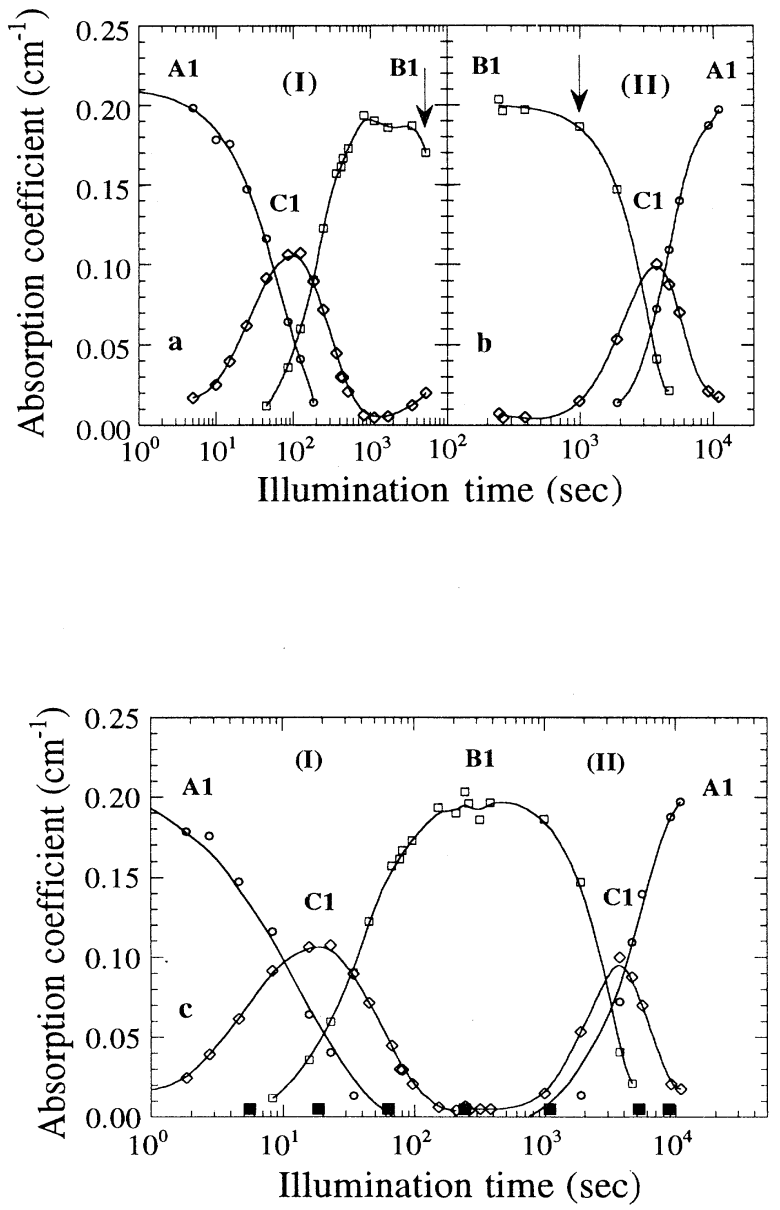

FIG. 2. Absorption coefficients of the three lines $A 1, B 1$, and $C 1$ change with illumination time in part $I$ of (a) and part II of (b), induced by $1.25-\mathrm{eV}$ illumination. Two arrows indicate the two similar intensity ratios in parts I and II; (c) the time scales in parts I and II are unified by multiplying a factor of 5.4 for that of II. Seven black square dots on abscissa are chosen as the typical illumination time points used for the photosensitivity measurement on the $\mathrm{XH}$ and $\mathrm{Fe}^{2+}$ centers shown in Fig. 4. 


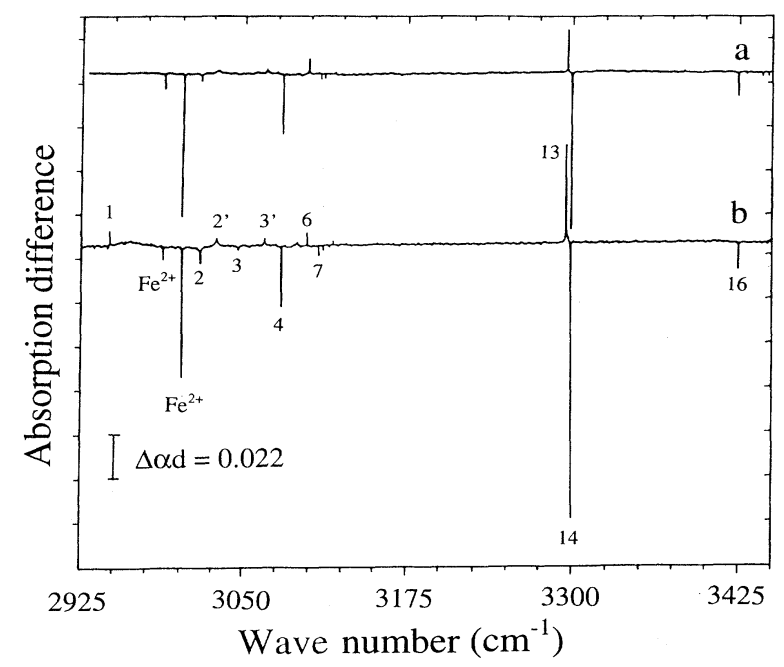

FIG. 3. Difference absorption spectra after and before (a) 150 $\min h v=1.25 \mathrm{eV}$ illumination and (b) $10 \mathrm{~min}$ white-light illumination on sample 1, at temperature $6 \mathrm{~K}$.

initiates the $(B \rightarrow C \rightarrow A)$ transfer discussed in the preceding section. This shows that during the $(A \rightarrow C \rightarrow B$ ) transfer, no intensity change of the LVM's discussed in this section takes place. As mentioned previously, ${ }^{17}$ a constant intensity ratio has been found between lines $X_{1}$ and 4, suggesting that they were two LVM's of the same center. This conclusion is strengthened by their photoinduced declining behavior during part II in the experiment. The photoinduced behavior of line 1 is more complicated. For short illumination times, its intensity decreases and a new line $\left(1^{\prime}\right)$ grows at $2791.8 \mathrm{~cm}^{-1}$. This is in qualitative agreement with the results of Ref. 14. For longer illumination time with $1.25-\mathrm{eV}$ photons there is no intensity difference observed (Fig. 5). But for 10min illumination with white light, the intensity of line 1 increases slightly (Fig. 3). However, this increase has not yet been correlated with the decrease of another LVM.

\section{Thermal recovery}

The permanent effects produced at $6 \mathrm{~K}$ by illuminating the SI GaAs sample with $1.25-\mathrm{eV}$ photons can be erased by raising the temperature of the sample under QTEC. This is shown in Fig. 6 for lines $X_{1}, 4$, and 14, which recover their pristine intensity at about $150 \mathrm{~K}$ (the annealing is for $20 \mathrm{~min}$ at each temperature). This recovery is correlated with the bleaching out of lines 6 and 13 .

\section{The weakly $n$-type sample}

In sample 6, after cooling under QTEC, $B$ is about twice more intense than $A$. After illumination with $1.25-\mathrm{eV}$ photon flux similar to that being used for parts I and II in the previous experiment for sample 1, the only result is the bleaching out of $A$ and the growth of $C$ with a final intensity comparable to that of $\boldsymbol{A}$ under QTEC. At the same time, after white-light illumination, known to speed up the process in the SI GaAs sample, a small decrease of the intensity of the $\mathrm{Fe}^{2+}$ lines is observed, but no intensity change of the LVM's discussed in Sec. III B is observed.

\section{DISCUSSION}

In this discussion, we consider the charge-transfer processes in the samples and the correlated kinetics behavior of the photosensitive centers. The charge transfer is

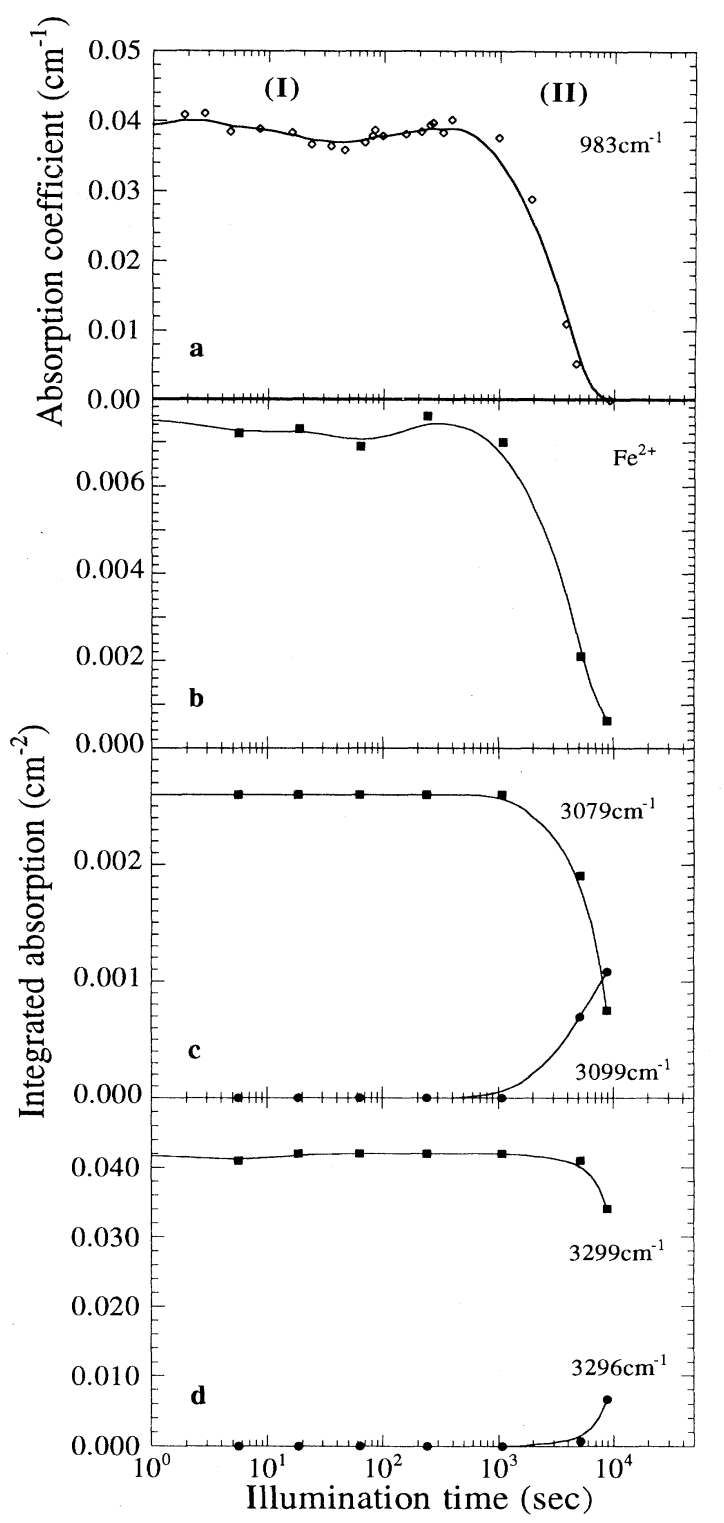

FIG. 4. (a) $1.25 \mathrm{eV}$ illumination-induced photoquenching of line $X_{1}$ at $983 \mathrm{~cm}^{-1}$ with the same time scale as that used for the measurement of $A, B$, and $C$; (b) photoquenching of the line associated with the $\mathrm{Fe}^{2+}$ center; (c) and (d) intensity decrease of lines 4 and 14, at 3079 and $3299 \mathrm{~cm}^{-1}$, respectively, and photoinduced new lines 6 and 13, at 3099 and $3296 \mathrm{~cm}^{-1}$, respectively, under the same illumination as used for measuring $A, B$, and $C$, recorded at the seven time points indicated in Fig. 3. 


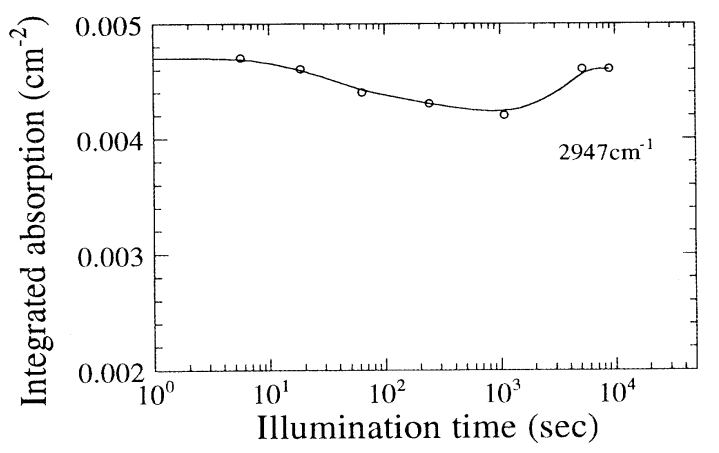

FIG. 5. Variation of the intensity of line $1\left(2947 \mathrm{~cm}^{-1}\right)$ under illumination, measured at the same time points as in Fig. 4.

detected by the intensity changes of the LVM's related to a given center, which are proportional to the changes of concentrations of the different charge states. We only summarize the situation for $\left(\mathrm{O} V_{\text {as }}\right)$ as it has been fully discussed in other papers. ${ }^{10,13}$ Three LVM's, $A, B$, and $C$, have been related to $\left(\mathrm{O} V_{\mathrm{As}}\right)^{0},\left(\mathrm{O} V_{\mathrm{As}}\right)^{2-}$, and $\left(\mathrm{O} V_{\mathrm{As}}\right)^{-}$,

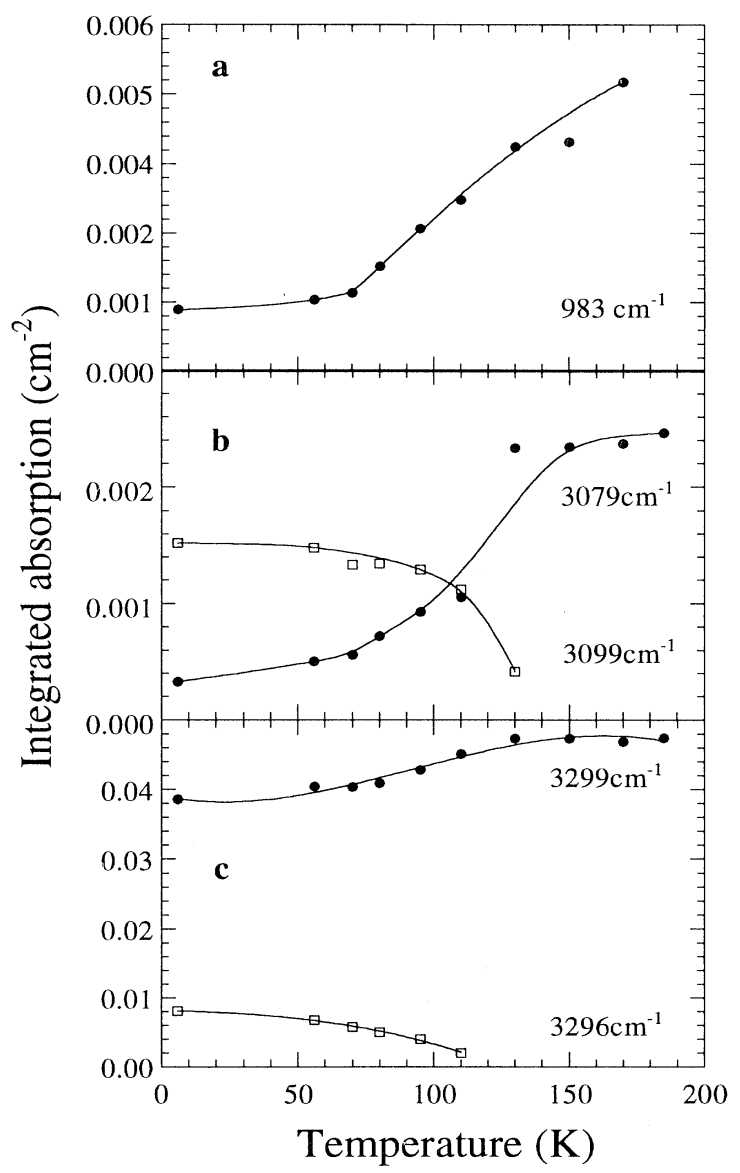

FIG. 6. Thermal recovery ( $\bullet$ ) of the photosensitive lines (a) $X 1\left(983.3 \mathrm{~cm}^{-1}\right)$, (b) $4\left(3079.2 \mathrm{~cm}^{-1}\right)$, and (c) $14\left(3299.95 \mathrm{~cm}^{-1}\right)$; also shown the corresponding annealing $(\square)$ of the photoinduced lines (b) $6\left(3099.08 \mathrm{~cm}^{-1}\right)$ and (c) $13\left(3296.4 \mathrm{~cm}^{-1}\right)$. Duration at each temperature is $20 \mathrm{~min}$. respectively. Process 1 , produced by illumination with photon $1.25 \mathrm{eV}$, and detected by the $(A \rightarrow C \rightarrow B)$ transfer is due to the capture of one electron by $\left(\mathrm{O} V_{\mathrm{As}}\right)^{0}$, giving $\left(\mathrm{O} V_{\mathrm{As}}\right)^{-}$, followed by the capture of one more electron by $\left(\mathrm{O} V_{\mathrm{As}}\right)^{-}$, giving $\left(\mathrm{O} V_{\mathrm{As}}\right)^{2-}$. Process 2 , detected by the $(B \rightarrow C \rightarrow A)$ transfer can result from the electron emission or hole capture by $\left(\mathrm{O} V_{\mathrm{As}}\right)^{-}$and $\left(\mathrm{O} V_{\mathrm{As}}\right)^{2-}$. A test of the actual mechanism is provided by the bleaching out of the $\mathrm{Fe}^{2+}$ lines, which can only be produced by a phototransfer from $\mathrm{Fe}^{2+}$ to $\mathrm{Fe}^{3+}$. As this occurs only in correspondence to process 2 , it clearly indicates hole capture, otherwise it would happen in correspondence to process 1 as well. It is very likely that the process is the same for $\left(\mathrm{O} V_{\text {As }}\right)$.

The fact that the photoinduced intensity changes on the presumably hydrogen related LVM's are produced under the same illumination conditions as part II for $\left(\mathrm{O} V_{\mathrm{As}}\right)$ and the photoionization of $\mathrm{Fe}^{2+}$ during that process seems to indicate that these changes are due to the same hole-capture process. The intensity decrease of the lines noted with $\mathrm{PI}^{-}$in Table $\mathrm{I}$ is attributed to the trapping of a hole by the related centers. When their concentration is high enough, the LVM's of the $\mathrm{OH}$ or $\mathrm{NH}$ modes modified by a different charge density are detected as new lines. The pairs of lines $(14,13),(4,6)$, and $\left(X_{1}, X_{2}\right)$, where the line whose intensity decreases is given first and followed by the corresponding emerging new line, illustrate this point (see Table I for the line labeling). A discussion of the microscopic nature of the corresponding defects can be found in Ref. 7. It has been shown that line 1 is related to another LVM at 1984.3 $\mathrm{cm}^{-1}$, both attributed to the stretch modes of two $\mathbf{H}$ atoms bonded to a substitutional $\mathrm{N}$ atom along a $\langle 111\rangle$ axis. ${ }^{14}$ Under illumination with $1.25-\mathrm{eV}$ photons, these two LVM's were reported to bleach out ${ }^{14}$ and new LVM's to appear at 2791.8 and $2042.5 \mathrm{~cm}^{-1}$. The lines at 1984.3 and $2791.8 \mathrm{~cm}^{-1}\left(1^{\prime}\right)$ have been observed in sample 1. However, in the absence of additional experimental data, it is difficult to speculate on the change of the charge state producing the decrease of the intensity of line 1 and the growth of the line at $2791.8 \mathrm{~cm}^{-1}$ for short illumination times and the increase of the intensity of line 1 for very long illumination times.

We have presented the effect of the photoinduced charge transfer on electrically active centers in SI GaAs containing $\mathrm{O}, \mathrm{N}$, and $E L 2$. This transfer is made possible by the presence of the $E L 2$ defect in the samples and its efficiency depends on the position of the Fermi level in the samples. The role of $E L 2$ in revealing the electrical activity of impurity centers in GaAs by acting under illumination in SI material, first as an electron source and then as a hole source, has attracted the attention in the early works ${ }^{4,5}$ and it can be described here as follows: In sample 1 , the Fermi level is pinned to the $E L 2^{0} / E L 2^{+}$ level at $E_{c}-0.8 \mathrm{eV}$, so that both $E L 2^{\circ}$ and $E L 2^{+}$are present under QTEC. Photons with $1.25-\mathrm{eV}$ energy can ionize electrons from $E L 2^{0}$ into the conduction band (process 1). The cross section $\sigma_{n}^{0}(1.25 \mathrm{eV})$ for this process is ${ }^{15}$ about $1.3 \times 10^{-16} \mathrm{~cm}^{-2}$ at $T=78 \mathrm{~K}$. It is assumed that slight difference of the photoionization cross sections at 78 and $6 \mathrm{~K}$ does not affect the validity of the 
analysis here. To explain the experimental observations, it must be assumed that $\left(\mathrm{O} V_{\mathrm{As}}\right)^{0}$ and $\left(\mathrm{O} V_{\text {As }}\right)^{-}$have a large capture cross section for the electrons at liquidhelium temperature (LHeT). Simultaneously, electrons can be photoemitted from the valence band to neutralize $E L 2^{+}$(process 2). The photoionization cross section for this process, notably, $\sigma_{p}^{0}$, as it can be considered as the photoionization of holes from $E L 2^{+}$into the valence band, is about one order of magnitude smaller than $\sigma_{n}^{0}$ for $1.25-\mathrm{eV}$ photons. ${ }^{15}$ This explains why the effect of the hole production due to EL 2 occurs only in part II of the illumination while the concentration of $E L 2^{+}$has been enhanced due to process 1 , i.e., electron photoionization from $E L 2^{0}$ plus electron capture into defect centers such as $\left(\mathrm{O} V_{\mathrm{As}}\right)$. The last photoinduced process is the transfer of normal $E L 2^{0}$ into the metastable state $E L 2^{*}$ with a cross section of $\sigma_{*}^{0}$ (process 3). At $1.25 \mathrm{eV}, \sigma_{*}^{0}$ is about $8 \%$ of $\sigma_{n}^{0} .{ }^{16}$ This is the process which gradually transfers the $E L 2^{0}$ state to the metastable $E L 2^{*}$ state. In the metastable state, which is the final state of $E L 2$ in the experimental procedure, neutral $E L 2$ is neither optically nor electrically active and it cannot retrap holes which are then available to the other hole traps. As said before, a signature of this trapping is the bleaching out of $\mathrm{Fe}^{2+}$ in sample 1 . If the concentration of $E L 2^{+}$available for process 2 came only from process 1 , the whole concentration of holes produced by process 2 would be used in part II of the illumination, as at the end of the interconversion, $\left(\mathrm{O} V_{\mathrm{As}}\right)^{0}$ is the same as at the beginning. The trapping of (additional) holes by $\mathrm{Fe}^{2+}$ and the $\mathrm{H}$-related centers can be explained only if $E L 2^{+}$is present in the sample under QTEC and this is an a posteriori proof that, in sample 1, the Fermi level is pinned to the $E L 2^{0} / E L 2^{+}$ level.

In sample 6, under QTEC $A$ and $B$ are simultaneously observed, and initially the intensity of $B$ is about twice that of $A$. According to the negative- $U$ model, the Fermi level at LHeT must be above the middle, in between the two thermal levels $\left(\mathrm{O} V_{\mathrm{As}}\right)^{2-} /\left(\mathrm{O} V_{\mathrm{As}}\right)^{-}$and $\left(\mathrm{O} V_{\mathrm{As}}\right)^{-} /\left(\mathrm{O} V_{\mathrm{As}}\right)^{0}$ which are reported at $E_{c} 0.58 \mathrm{eV}$ and $E_{c} 0.14 \mathrm{eV}$, respectively. ${ }^{8,9}$ Therefore only $E L 2^{0}$ is initial- ly present. During the illumination, $\left(\mathrm{O} V_{\mathrm{As}}\right)^{0}$ is totally converted into $\left(\mathrm{O} V_{\text {As }}\right)^{-}$by process 1 . The reason why the interconversion observed in sample 1 is not observed in sample 6 can eventually be explained by the shorter lifetime of the minority carriers (holes) in the $n$-type material, preventing the trapping of a second electron by $E L 2^{+}$. Also, the total number of $E L 2^{+}$available for process 2 is smaller in this sample, as initially there are no $E L 2^{+}$. In that case, process 1 can compete with process 2 and direct electron-hole recombination may play a significant role. Consequently, there are neither excess electrons nor holes to make the interconversion of either $\left(\mathrm{O} V_{\text {As }}\right)^{-} \rightarrow\left(\mathrm{O} V_{\text {As }}\right)^{2}$ or $\quad\left(\mathrm{O} V_{\text {As }}\right)^{-} \rightarrow\left(\mathrm{O} V_{\text {As }}\right)^{0}$ occur. Therefore the ending status of $\left(\mathrm{O} V_{\text {As }}\right)$ in sample 6 after the illumination is $\left(\mathrm{O} V_{\mathrm{As}}\right)^{2-}$ and $\left(\mathrm{O} V_{\mathrm{As}}\right)^{-}$, i.e., only $B+C$ appear in the infrared absorption spectrum.

\section{CONCLUSION}

It has been shown that the photoinduced charge changes of $\left(\mathrm{O} V_{\text {As }}\right)$ and of H-related defects in SI GaAs illuminated with $1.25-\mathrm{eV}$ photons were due to the pinning of the Fermi level to the $E L 2^{0} / E L 2^{+}$level, to the amphoteric behavior of $E L 2$, and finally to the metastability of $E L 2^{\circ}$. This results in the SI GaAs sample, in a nonreversible cyclic change of the charge states and to the evidence of electrical activity of some $\mathrm{H}$-related c_. rs If the Fermi level is not pinned to the $E L 2^{0} / E L 2^{+}$level, different but similar photosensitivity of those centers can also be observed, due to the sample amphoteric and metastable behavior of EL2.

\section{ACKNOWLEDGMENTS}

We are grateful to B. Clerjaud for stimulating discussions, and to C. Naud and C. Porte for technical assistance. We thank Y. H. Wang and B. C. Ma for kindly providing the samples. C. Y. S. and W. K. G. are grateful to the DAG 93/94 Grant No. SC-16 from HKUST.
${ }^{1}$ C. Y. Song, W. K. Ge, D. S. Jiang, and C. C. Hsu, Appl. Phys. Lett. 50. 1066 (1987).

2J. Schneider, B. Dischler, H. Seelewind, P. M. Mooney, J. Lagowski, M. Matsui, D. R. Beard, and R. C. Newman, Appl. Phys. Lett. 54, 1442 (1989).

${ }^{3}$ H. Ch. Alt, Appl. Phys. Lett. 54, 1445 (1989).

${ }^{4}$ H. Ch. Alt, Appl. Phys. Lett. 55, 2736 (1989).

${ }^{5}$ C. Y. Song, B. Pajot, and C. Porte, J. Appl. Phys. 67, 7307 (1990).

${ }^{6}$ C. Y. Song and B. Pajot, Phys. Rev. B 41, 12330 (1990).

${ }^{7}$ B. Pajot and C. Y. Song, Phys. Rev. B 45, 6484 (1992).

${ }^{8}$ H. Ch. Alt, Phys. Rev. Lett. 65, 3423 (1990).

${ }^{9}$ M. Skowronski, S. T. Neild, and R. Kremer, Appl. Phys. Lett. 57, 902 (1990).
${ }^{10}$ C. Song, B. Pajot, and C. Porte, in 21st International Conference on the Physics of Semiconductors, edited by P. Jiang and H. Z. Zheng (World Scientific, Singapore, 1992), p. 1629.

${ }^{11}$ G. K. Ippolitova and E. M. Omel'yanovskii, Fiz. Tekh. Poluprovodn. 9, 236 (1975) [Sov. Phys. Semicond. 9, 156 (1975)].

${ }^{12}$ G. M. Martin, Appl. Phys. Lett. 39, 747 (1981).

${ }^{13}$ H. Ch. Alt, Mater. Sci. Forum 143-147, 283 (1994).

${ }^{14}$ W.-S. Hahn, B. Clerjaud, D. Côte, F. Gendron, C. Porte, W. Ulrici, D. Wasik, and W. Wilkening, Mater. Sci. Forum 143147, 277 (1994).

${ }^{15}$ P. Silverberg, P. Omling, and L. Samuelson, Appl. Phys. Lett. 52, 1689 (1988).

${ }^{16}$ G. Vincent, D. Bois, and A. Chantre, J. Appl. Phys. 53, 3643 (1982). 